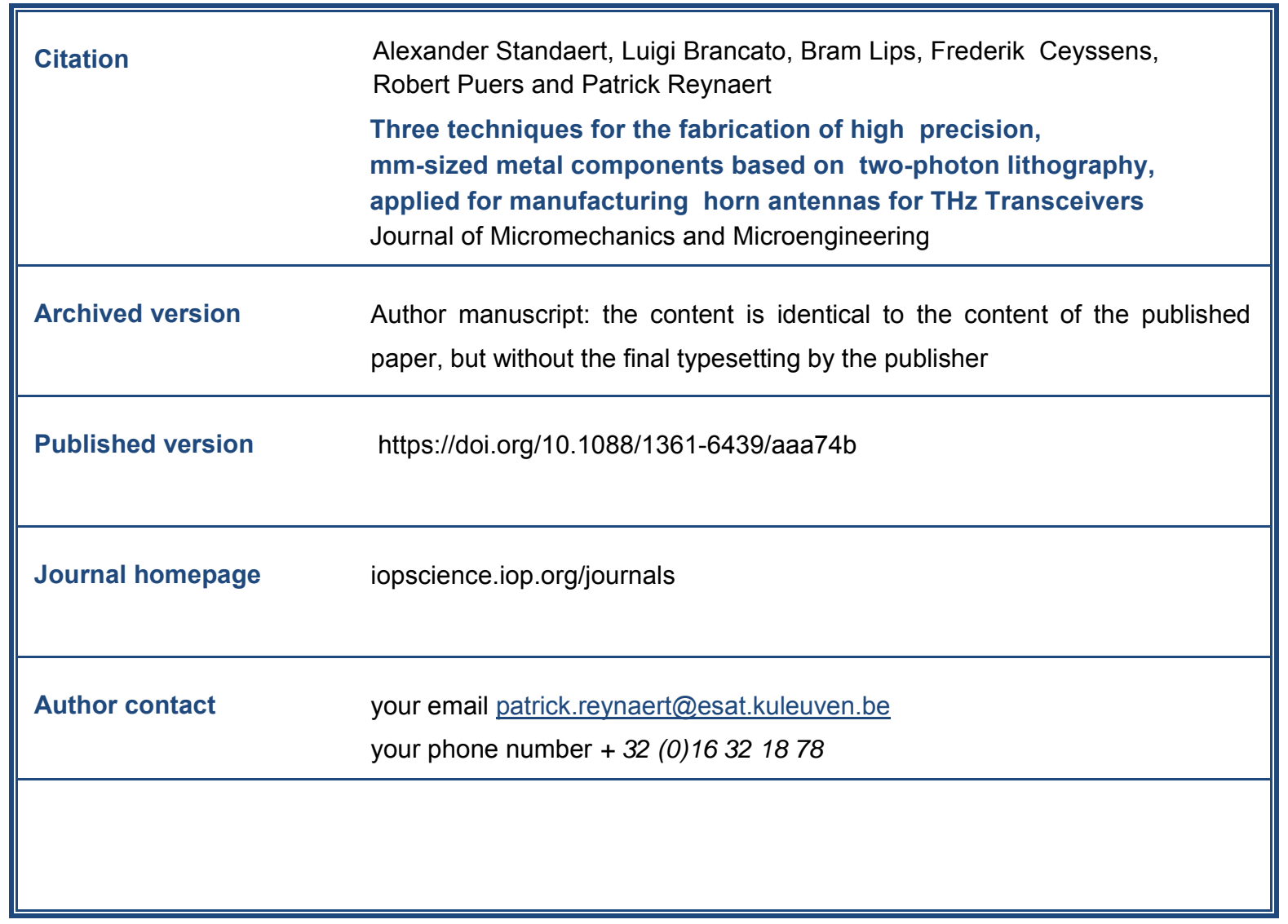

(article begins on next page) 


\title{
Three techniques for the fabrication of high precision, mm-sized metal components based on two-photon lithography, applied for manufacturing horn antennas for $\mathrm{THz}$ Transceivers
}

\author{
Alexander Standaert, Luigi Brancato, Bram Lips, Frederik \\ Ceyssens, Robert Puers and Patrick Reynaert \\ Kasteelpark Arenberg 10, Heverlee B-3001, Belgium \\ E-mail: astandae@esat.kuleuven.be \\ September 2017
}

\begin{abstract}
This paper proposes a novel packaging solution which integrates micromachined 3D horn antennas with millimeter-wave and $\mathrm{THz}$ tranceivers. This packaging solution is shown to be a valid competitor to existing technologies like metallic splitblock waveguides and low temperature cofired ceramics. Three different fabrication methods based on two-photon lithography are presented to form the horn antennas. The first uses two-photon lithography to form the bulk of the antenna. This structure is then metalised through physical vapor deposition (PVD) and copper plating. The second fabrication method makes use of a soft polydimethylsiloxane (PDMS) mold to easily replicate structures and the third method forms the horn antenna through electroforming. A prototype is accurately positioned on top of a $400 \mathrm{GHz} 28 \mathrm{~nm}$ CMOS transmitter and glued in place with epoxy, thus providing a fully packaged solution. Measurement results show a $12 \mathrm{~dB}$ increase in the antenna gain when using the packaged solution. The fabrication processes are not limited to horn antennas alone and can be used to form a wide range of mm-sized metal components.
\end{abstract}

Keywords: Horn antenna, THz, packaging, two-photon lithography, replica molding, electroforming

\section{Introduction}

In the field of integrated circuits, there is a growing trend to design systems at higher frequencies. These systems have interesting applications in spectroscopy and imaging [1][2][3][4]. Recently multiple examples have been shown in research of $\mathrm{THz}$ systems $(300 \mathrm{GHz}-3 \mathrm{THz})$ designed in bulk CMOS technologies, as opposed to systems designed in the more expensive III-V based technologies, thus paving the way for cheap consumer products [2][3]. However, the packaging of these chips is still a very costly process with many design and fabrication challenges [5]. In the literature and industry, several 
packaging solutions are used: metallic split-block waveguides [6][7], low temperature cofired ceramics (LTCC) [8][9] and silicon lenses [10][11]. The metallic split-block waveguides are made through CNC milling and are currently considered the most reliable packaging method. As frequency increases however, dimension and alignment tolerances increase dramatically, hence also the cost. The use of LTCC technology is an upcoming and promising $\mathrm{THz}$ packaging solution. This technology makes use of a stack ceramic substrates which are laminated together, forming 2.5D waveguide and transmission line structures. The downside to this technology is the manufacturing tolerances and large signal loss [5]. The third and final commonly used packaging technique relies on high resistivity silicon lenses. These lenses are usually mounted at the backside of a silicon die, greatly improving the directivity of on-chip antennas. The lenses have to be precision ground to shape, with a process not very suitable for mass production [12].

This paper explores the use of micro-machined horn antennas as a packaging solution for a $400 \mathrm{GHz} 28 \mathrm{~nm}$ CMOS transmitter. Section 2 describes the structure of the packaged system and addresses the mechanical design aspects of the the horn antenna. In section 3, three different fabrication methods are proposed. Each process is discussed in detail along with its advantages and limitations. The first process makes use of two-photon lithography to 3D print the bulk of the antenna, after which a metal coating is applied through PVD and copper plating. In the second fabrication method, the mechanical structure is realized by vacuum casting an epoxy in a PDMS mold followed by a metallization step. The third and final fabrication method uses electroforming to grow a copper horn antenna on an aluminum coated 3D printed mandrel. Section 4 describes how the fabricated horn antennas are mounted on top of the CMOS transmitter. Finally, an dimensional error analysis and the measurements of the fully packaged system are shown in section 5 along with a discussion of the results.

\section{System Overview and Mechanical Design}

An schematic drawing of the full $400 \mathrm{GHz}$ system can be seen in Fig 1a. It consists of a CMOS transmitter with an on-chip radiating source under the form of a dipole antenna. To boost the antenna gain, a micromachined horn antenna is put directly on top of the chip. The horn antenna will pick up the radiated waves from the on-chip dipole and focus them in one direction. Two sections are distinguishable in this antenna. At the bottom a square section segment acts as a waveguide feed. This then transitions in a tapered horn segment (Figure 1b). The electrical design of the packaged system is not the focus of this paper. However, several electrical aspects should be taken in account during the mechanical design. Firstly, the horn antenna should have minimal interference with the passive microwave components on the chip. This translates itself in the requirement that the wall thickness for the waveguide feed should be kept as thin as possible. A thin layer, however, will result in a very fragile structure that can be easily damaged during the mounting of the horn antenna on the chip (Section 4). In this case the wall thickness at the waveguide feed is around $100 \mu \mathrm{m}$. A second mechanical 


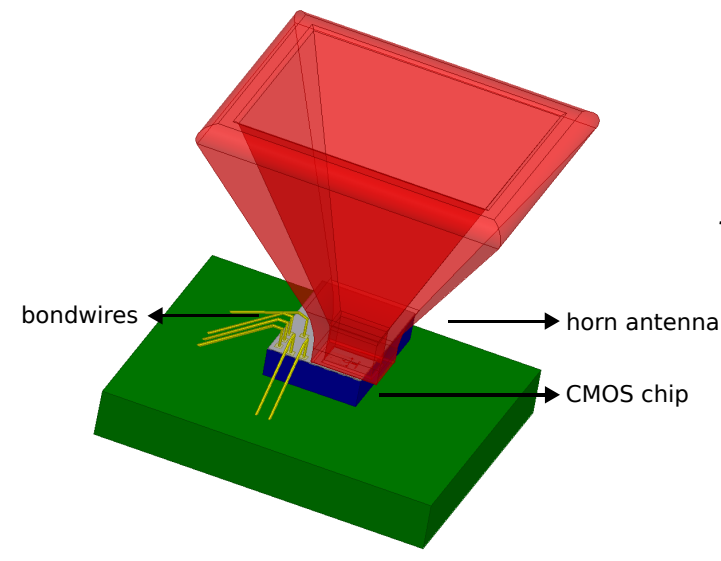

(a) Overview of the packaged system

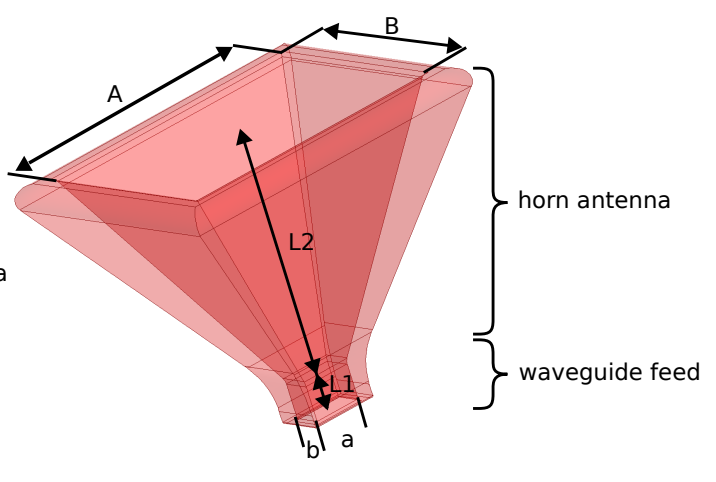

(b) Parameters of the horn antenna

Figure 1

issue is the location of the bondwires around the chip. The antenna body should be far enough from the bondwires to prevent short circuits. The waveguide length (L1) should be long enough to ensure a clearance between the horn antenna and the bondwires. On the other hand, making L1 too long will cause step coverage problems during the metalization process. A third aspect that has influence on the mechanical design is the length of the horn (L2). From an electrical standpoint, more antenna gain will be achieved the longer L2 becomes, assuming A and B are scaled accordingly [13]. A long L2, however, will greatly increase the print time with two-photon lithography process. Also the Nanoscribe machine has limits to its maximum achievable print volume, as will be explained in the next section. One could argue to use a SLA or DLP printing process instead of a two-photon lithography printing process. However, these processes have a too low resolution to meet the roughness specification the horn antenna requires. Table 1 shows the main dimensions for the prototypes presented in the next sections.

Table 1: Main dimensions of the different prototypes

\begin{tabular}{llll}
\hline Parameter & $\begin{array}{l}\text { Prototype two-photon } \\
(\text { section 3.1) }\end{array}$ & $\begin{array}{l}\text { Prototype replica mold- } \\
\text { ing (section 3.2) }\end{array}$ & $\begin{array}{l}\text { Prototype electroform- } \\
\text { ing (section 3.3) }\end{array}$ \\
\hline L1 & $400 \mu \mathrm{m}$ & $200 \mu \mathrm{m}$ & $400 \mu \mathrm{m}$ \\
$\mathrm{L} 2$ & $2440 \mu \mathrm{m}$ & $1950 \mu \mathrm{m}$ & $2400 \mu \mathrm{m}$ \\
$\mathrm{A}$ & $2760 \mu \mathrm{m}$ & $1970 \mu \mathrm{m}$ & $2740 \mu \mathrm{m}$ \\
B & $2160 \mu \mathrm{m}$ & $1970 \mu \mathrm{m}$ & $2120 \mu \mathrm{m}$ \\
$\mathrm{a}$ & $300 \mu \mathrm{m}$ & $300 \mu \mathrm{m}$ & $320 \mu \mathrm{m}$ \\
$\mathrm{b}$ & $500 \mu \mathrm{m}$ & $300 \mu \mathrm{m}$ & $600 \mu \mathrm{m}$ \\
\hline
\end{tabular}




\section{Fabrication Process}

In two photon lithography[14], a femtosecond pulsed infrared laser is focused in a relatively thick layer of UV-sensitive material. At the point of focus, the intensity is so high that two infrared photons essentialy get absorbed at the same moment, locally yielding the same effect as a single UV photon. This is a highly nonlinear effect: any point above or below the point of focus is receiving to enough light and will not crosslink at all[15]. Thus, freeform 3D structures can be written making it an ideal tool for our application[16][17].

\subsection{Fabrication Process through Two-photon Lithography}

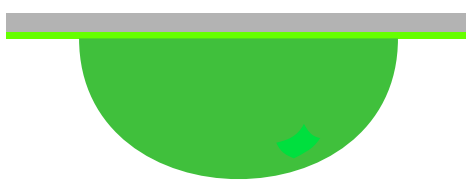

(a)

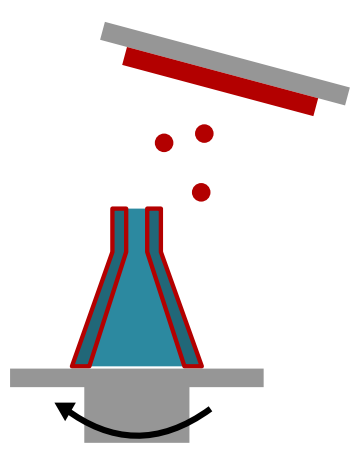

(d)

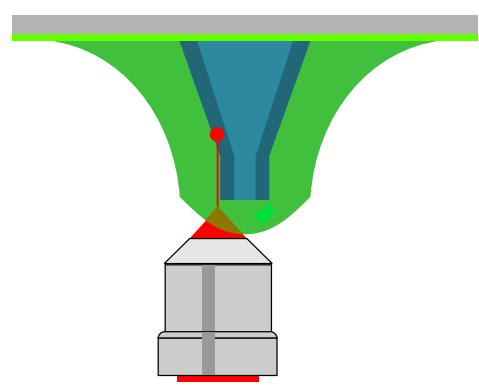

(b)

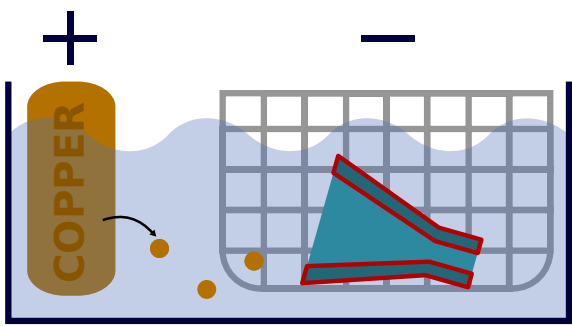

(e)

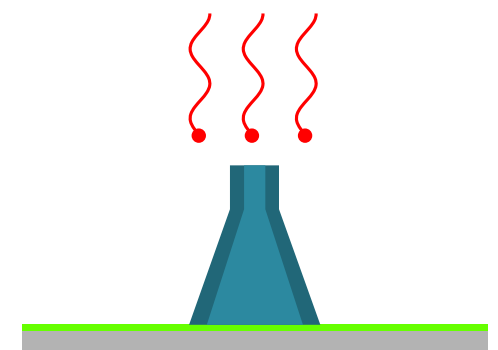

(c)

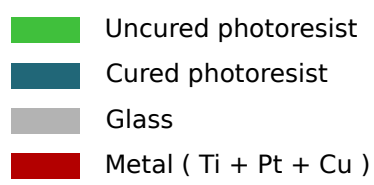

Figure 2: Schematic summary of the fabrication process using two-photon lithography: (a) substrate preparation. (b) two-photon lithography. (c) development and curing. (d) applying PVD seed layer. (e) copper plating.

A first prototype was made by printing the structural form of the horn antenna using a commercial 3D direct laser writing system from Nanoscribe GmbH $\ddagger$ (Photonic Professional Gt). This system can be configured to work as an immersion lithography or Dip-in lithography system[18]. In the former, an in oil immersed lens writes structures 
in resist, located on the topside of a glass substrate. This has the disadvantage that the z-range is very limited. In the Dip-in configuration, the lens is immersed in the resist which is located at the bottomside of a glass substrate (Figures 2a-2b). It would seem that the maximal printable height is now only limited by the range of the z-stage. In practice, however, as the system is gravity fed, the size of the printable structure is limited by the droplet of photoresist. If the volume of the initial droplet is too small, the finished print will be incomplete and contain voids where the laser was printing in air. If the volume of the initial droplet is too big, the photoresist will have an insuficient amount of surface tension to stay put and it will leak away resulting in the same problem. Experimentally, it was determined that the used setup is capable of handling a print volume of about $3 x 3 x 3 \mathrm{~mm}$ when using Ip-Dip as photoresist. IP-Dip from Nanoscribe $\mathrm{GmbH}$ is a negative-tone photoresist similar to SU8 but optimized for high resolution two-photon lithography[19].

When printing a large structure, printing time becomes an issue. This is remedied by only printing an outer shell of $12 \mu \mathrm{m}$ and some support scaffolding. The triangular scaffolding is placed inside of the structure at intervals of $40 \mu \mathrm{m}$. This reduces the print time to a reasonable 10-13 hours. Figure 3a shows the scaffolding during the printing process. The structures were printed using a lens with a $25 \mathrm{x}$ magnification.

The fabrication process is as follows: To enable the release of the prototype from the glass substrate, a thin layer of LOR10B resist is applied. After printing, the sample is developed for about 40min in PGMEA and rinsed with IPA and DI water. The structure is then placed under a $400 \mathrm{~nm}$ flood lamp for 10min, to expose all the uncured photoresist trapped in the scaffolding mesh, thus resulting in a solid part (Figure 2c). The shrinkage of the part caused by the flood lamp will be further examined in section 5.1. The layer of LOR10B is then dissolved in a mixture of 351 developer and DI water (1 to 4 volume ratio), thus releasing the printed part from the glass support substrate. Next, a layer of Ti and Pt are applied through PVD sputtering as seed layer for the next electroplating step. This step was repeated twice and the part was flipped around in-between to ensure complete coverage of the irregular shape. The target of the PVD machine is set at an angle with reference to a rotating table to provide good coverage. This setup makes it possible to also metalize the sides of the waveguide feed with no step coverage problems. The samples were sputtered at $200 \mathrm{~W}$ for $45 \mathrm{~min}$ at $40 \mathrm{sccm}$. Although it was not possible to measure the thickness of the PVD layer on the 3D-printed part, the same metalisation step on a glass wafer set at the same distance resulted in a total thickness of $200 \mathrm{~nm}$. It should be pointed out that the seed layer could just as well be a combination of $\mathrm{Cr}$ and $\mathrm{Cu}$ but these metals were not allowed in the used PVD machine due to contamination reasons. Next the samples are electroplated in a bright copper electrolyte from TIFOO. Given the fragile nature of the samples, the cathode could not be directly clamped to them. A metal basket was developed (Figure 3b) in which the samples can be placed during plating. Copper was plated for $15 \mathrm{~min}$ at $300 \mathrm{~mA}$ yielding a copper thickness of $5 \mu \mathrm{m}-7 \mu \mathrm{m}$. This was measured on a seperate glass slide that was jointly put in the plating basket. Finally, the plated parts were thoroughly rinsed with 
DI water and dried on a hotplate. Figure 3c illustrates the finished prototype.

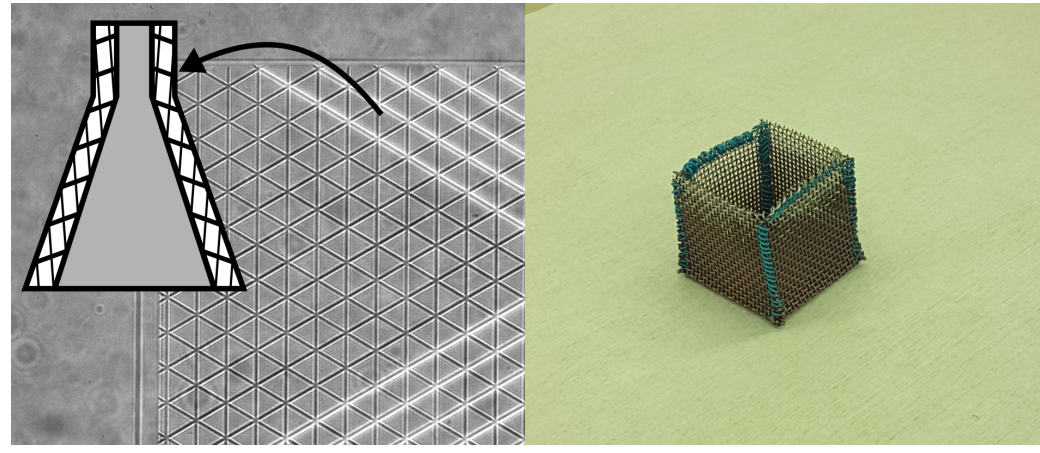

(a) Scaffolding inside of the horn antenna as seen during the two-photon lithography process (b) Metal basket used for copper plating

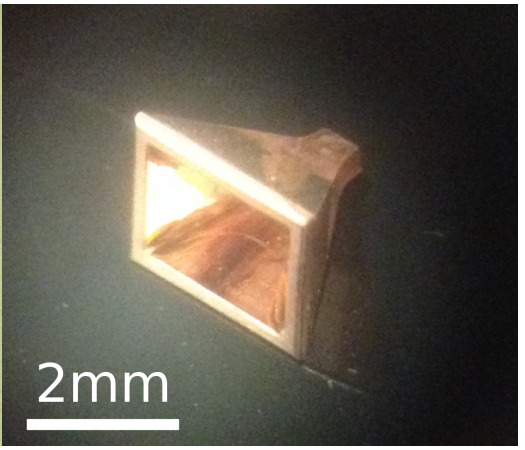

(c) Finished horn antenna

Figure 3

\subsection{Fabrication process through replica molding}

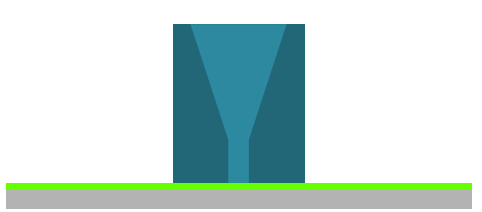

(a)

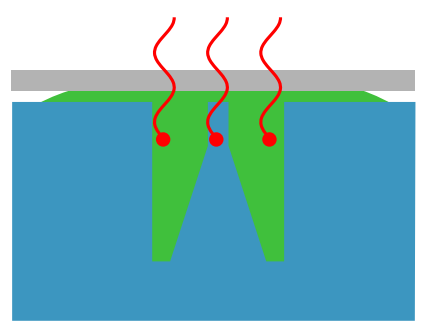

(d)

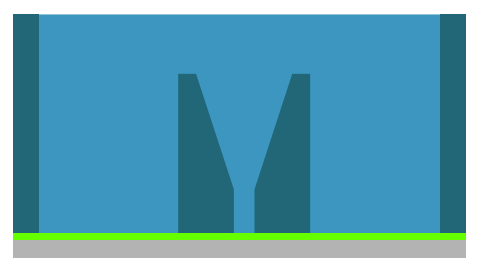

(b)

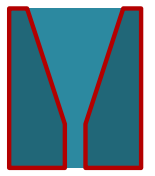

(e)

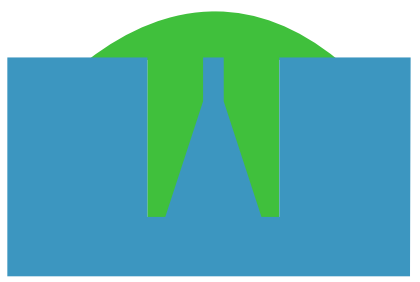

(c)

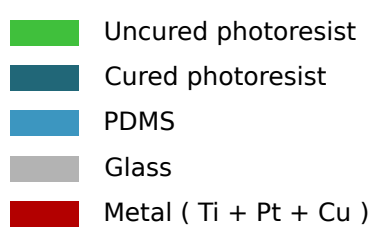

Figure 4: Schematic summary of the fabrication process using replica molding: (a) fabrication of mold master using two-photon lithography. (b) casting of the PDMS mold. (c) casting and degassing of Ip-Dip replica. (d) removal of excess photoresist and UV curing. (e) metalisation of the replica.

One of the issues with the fabrication process in the previous section, is the long print time of the two-photon lithography step. A remedy for this could be using replica 
molding[20][21]. For this to work, the design was altered such that the part could be made in a one piece mold. The design now looks like a cube with a piramid subtracted from it (Figure 5a). This results in the same electric performance for the horn antenna itself, but the base of the horn antenna does take more space which would have to be taken into account when placing the bondpads on the CMOS chip. A master part was printed using two-photon lithography (Figure 4a). A two component PDMS (Silgard 184) was mixed up, degassed and poured over the master. After degassing under a pulsating vacuum, the PDMS is cured at $65^{\circ} \mathrm{C}$ for 8 hours (Figure $4 \mathrm{~b}$ ). The adhesion between the PDMS and the glass/Ip-Dip is not very good, so no release agent was needed to demold the master part. Several different epoxies were tried to do the replica molding. A good epoxy should be not too viscous so that it can fill the small voids of the mold under the vacuum. e.g SU8 could be a good candidate, however the vacuum step causes the solvent to expand and to evaporate, resulting in a very viscous material with many entrapped bubbles. Some conductive epoxies were also tested out but the are already viscous from the start and cure too quick before it is properly degassed. Eventually Ip-dip was chosen as the best candidate. This photoresist has a reasonable viscosity but has the benefit that its not heat curable but it is UV curable. The mold could thus be heated up during degassing, lowering the viscosity and easing the removal of entrapped air bubbles. Figure 5b shows the PDMS mold together with a SU8 and Ip-Dip replica. Notice the small bubbles still remaining in the SU8 replica. After degassing, a glass wafer was placed on top of the mold. The weight of this wafer was sufficient to squeeze out any excess epoxy out of the mold (Figure 4c). The mold is then cured by illuminating through the glass wafer after which the glass wafer is removed. During the removal of the glass wafer, the small rim of excess epoxy or scum just breaks off, creating the desired replica. The horn antenna prototype is then metalised using the same procedure as described in the previous section. Figure 5c shows the finished prototype.

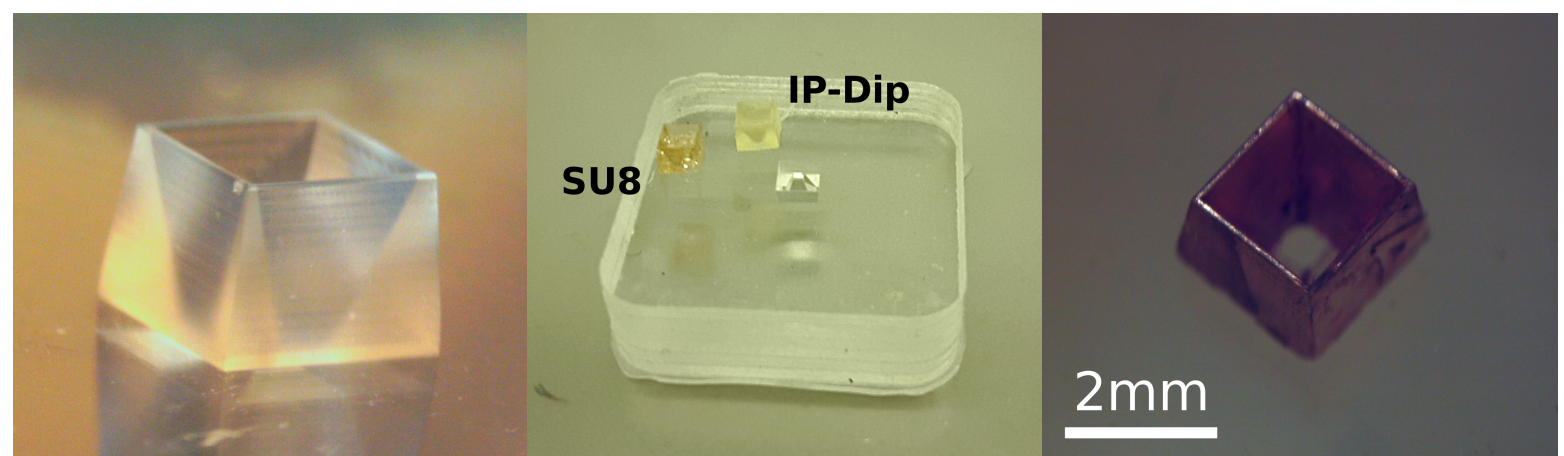

(a) 3D printed master

(b) PDMS mold with Ip-Dip and SU8 replicas

(c) Finished horn antenna

Figure 5 


\subsection{Fabrication process through electroforming}

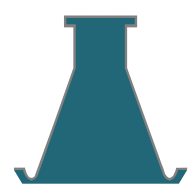

(a)

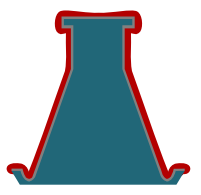

(b)

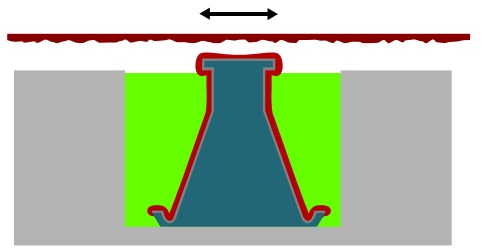

(c)

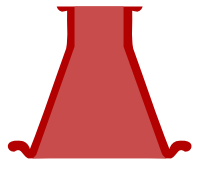

(d)

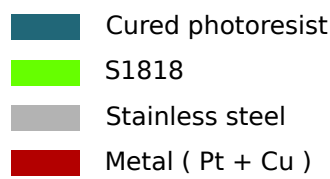

Figure 6: Schematic summary of the fabrication process using electroforming: (a) fabrication of the mandrel using two-photon lithography. (b) apply PVD layer titanium, aluminium and platinum. Then electroplate copper. (c) Removal of the overplating with abrasive paper in a jig. (d) Dissolve the aluminium release layer and removal of the mandrel.

In sections 3.1 and 3.2 , the horn antennas were built up of a polymer body with a thin metallic coating. Although the 3D direct laser writing of the antenna body allows great flexibility to the shape, this flexibility is limited through the directive metalisation of the PVD seed layer. Electroforming is a process where a thick layer of copper or nickel is plated around a conductive mold or mandral[22]. This mandral is afterwards removed or dissolved. It has traditionally been used to create waveguide components where it is easier to machine the inside form than to hollow out a part precisely [23][24][25]. From an electrical point of view, only the inner side of the horn antenna is important. An inner mold is therefore printed with two-photon lithography (Figure 6a). This is subsequently coated first with titanium, then with a $100 \mathrm{~nm}$ thick layer of aluminum and finally with platinum. The aluminum layer will be used as release layer of the mold but has the problem that it does not serve as an appropriate seed layer for the used acid copper plating bath. This could be remedied with a copper cyanide plating bath[26] but adding the thin layer of platinum was considered a safer solution. This mandral is then plated for 4 hours using $400 \mathrm{~mA}$ of current. Given the long plating time and the fact that plating was done in a metal basket, the part had to be flipped around every so often to prevent stiction to the metal basket. The resulting copper layer was between $100 \mu \mathrm{m}$ and $150 \mu \mathrm{m}$. One issue with plating thick metal layers is overplating. This was most prominent at the waveguide feed. An additional step is required to clean 
up the waveguide feed of the horn antenna (Figure 6c). The horn antenna is temporary fixed in-place in the cavity of a metal holder with some S1818. The topside of the horn antenna is then ground flat with some fine abrasive paper. The metal holder provides a natural stopping point during the grinding and ensures a nice parallel flange. Next, the aluminum sacrificial layer was left to dissolve in sodium hydroxide. The dip in Sodium hydroxide, causes the structure to swell. Rinsing it in DI water, and subsequently letting it dry under continuous air flow, allows the device to de-swell, and to release from the mould. Finally the horn antenna is electroplated for a second time to also cover the platinum layer at the inside with copper. Figure $7 \mathrm{c}$ shows the finished prototype.

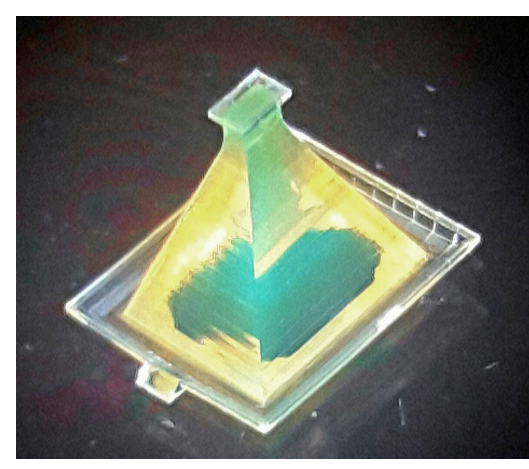

(a) 3D printed mandrel before applying seed layer

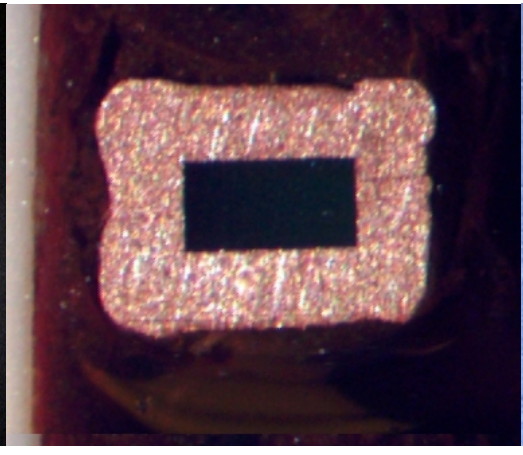

(b) Opening of the waveguide feed after grinding flat

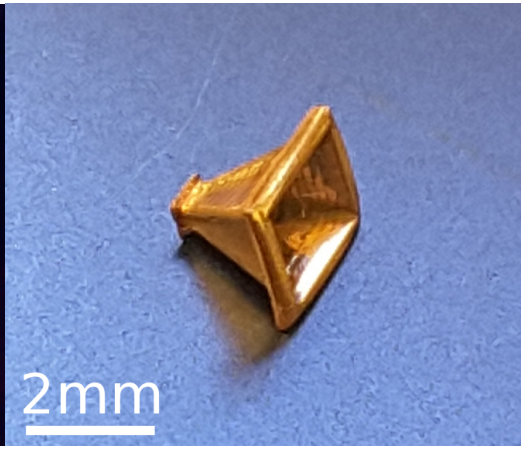

(c) Finished prototype

Figure 7

\section{Antenna Integration}

As shown in figure 1a, the horn antenna was designed to be placed on top of a $400 \mathrm{GHz}$ CMOS transmitter with an on-chip antenna. The alignment is very critical as any mis-alignments results in loss in coupling efficiently. For this application a placement accuracy of about $10 \mu \mathrm{m}$ is required. The alignment and placement is done with a Finetech FINEPLACERß. Easy alignment of the waveguide feed with references on the CMOS chip can be done through an overlay vision alignment system. A horn antenna made with the method described in section 3.1 is attached on a piece of $70 \mu \mathrm{m}$ thick glass with S1818 positive photoresist. This glass substrate provides a clean and smooth surface for the vacuum probe of the fineplacer to grab on to. With the fineplacer, the waveguide of the horn antenna can now be aligned and flipped on top of the CMOS chip as can be seen in figure 8. Epotek H54 epoxy can now carefully be applied to glue the horn antenna to the chip and shield of the bondwires. The hotplate in the fineplacer is used to cure the epoxy at $110^{\circ} \mathrm{C}$ for 1 hour. At this point the temporary glass substrate can be removed by dissolving the S1818 in acetone, leaving a fully packaged result. 


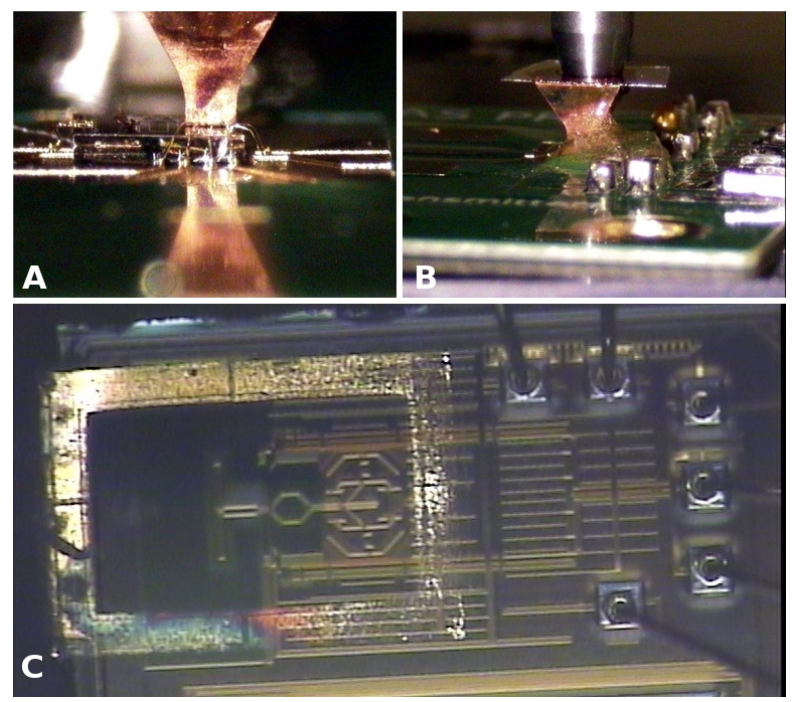

Figure 8: Positioning and glueing of the horn antenna on the chip. A piece of glass is temporary glued to the horn antenna so the vacuum probe can hold on to it . After alignment, the horn antenna is glued into place with epoxy (B). The horn antenna and chip are aligned using an overlay vision alignment system. The on-chip antenna is aligned with the waveguide feed $(\mathrm{C})$. The bondwires stay clear from the horn antenna (A) and (C)

\section{Results and Discussion}

\subsection{Fabrication accuracy}

The presented fabrications processes will naturally exhibit errors compared to the initial design. This section will discuss the accuracy of the fabricated prototypes and the causes of errors. First the errors in lateral dimensions are discussed after which the vertical dimensions are discussed. The waveguide feed is the only feature that has perfectly square sharp corners from which the lateral dimensions are easily measurable. The inside dimensions of the waveguide feed were therefore chosen for evaluation of size. The measurements were done under a digital microscope with a 200x magnification. Both the width (a) and height (b) of the measured waveguide feed had decreased in size. This shrinkage compared to the designed values is illustrated in table 2 . The shrinkage is defined as $\Delta L / L_{0}$.

There are several effects that could contribute to the decrease in lengths. For the prototypes of sections 3.1 and 3.2, copper is electroplated at all sides of the polymer structure. No compensation was done to the structure, to account for the copper layer which is assumed to be about $10 \mu \mathrm{m}$ thick.

A second cause of errors, which is applicable to all prototypes, is the shrinkage of the polymer itself. This can happen during the curing of the Ip-Dip under the flood lamp. In this stage, the outer shell together with some scaffolding are already fully cured. It is therefore expected that the scaffolding density will have an effect on the amount of 
Table 2: Shrinkage of the waveguide feed for the different prototypes

\begin{tabular}{|c|c|c|c|}
\hline & shrinkage a $(\%)$ & shrinkage b $(\%)$ & shrinkage $\mathrm{H}(\%)$ \\
\hline prototype two-photon & $4.6-5.4$ & $4.3-5.6$ & $0.92-3.13$ \\
\hline prototype molding master & 3.6 & 4.0 & 1.45 \\
\hline replicas & $5.3-6.6$ & $4.6-6.6$ & $0.9-2.5$ \\
\hline prototype electroforming & 6.5 & 6.5 & 2.75 \\
\hline
\end{tabular}

shrinkage. To investigate this effect, some test cubes with a side length of $300 \mu \mathrm{m}$ were printed. The outer skin of the cubes was $12 \mu \mathrm{m}$ and triangular scaffolding were placed at distances ranging from $20 \mu \mathrm{m}$ to $100 \mu \mathrm{m}$. Additionally a fully printed and a hollow scafoldless cube were also made. Figure 10 shows the different test structures. Notice how the hollow test structure shows signs of deformation due to insufficient support. The shrinkage of the cubes was measured after curing under a flood lamp for 10min and the results are shown in Figure 10. Clearly there is more shrinkage when the density of the scaffolding is reduced. The shrinkage of the $40 \mu \mathrm{m}$ scaffolding test case corresponds nicely with shrinkage of the fabricated prototypes where $40 \mu \mathrm{m}$ scaffolding was also used. Although the shrinkage in the polymer could be prevented by increasing the intensity of the scaffolding, a more print-time friendly solution would be to scale the design to compensate the shrinkage.

Finally for the prototypes made through replica molding, undergo distortion during three distinct fabrication steps. Firstly there is some shrinkage during the curing of the master part. Secondly there are inaccuracies during the forming of the PDMS mold and finally there is shrinkage of the IP-Dip during the replication. In Table 2 one can clearly see the increase in dimensional errors between the master and replicas.

The heights of all prototypes were also measured to evaluate errors in vertical dimensions. The heights were measured with a Zeiss Axio Imager M1 microscope. The Z-stage of the microscope is zeroed while being focused on the holding platform afterwards the height is readout when the focus is moved to the top-side of the horn antenna. A lens with 50x magnification was used to minimize dimensional uncertainties caused by the focus range. As can be seen in Table 2, the shrinkage in the height for all prototypes is between 1 and 3\%. All prototypes were printed with a layer spacing of $1.5 \mu \mathrm{m}$. Interestingly enough, some of the prototypes made with the replica molding process, show a smaller shrinkage then the master part. A possible explanation for this could be that during the removal of the excess photoresist (Process step described in Figure 4d), some scum remains essentially increasing the height compared to the master. 


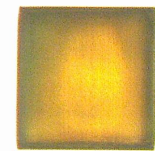

Full

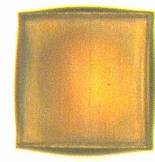

Hollow

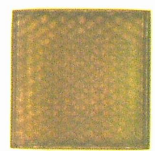

20um hatching

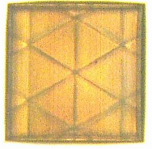

100um hatching

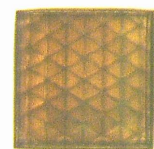

40um hatching

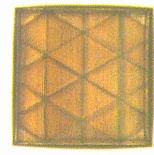

80um hatching

Figure 9: Test case to determine the relationship between scaffolding densities and the total amount of shrinkage. $300 \mu \mathrm{m}$ cubes are with an outer skin thickness of $12 \mu \mathrm{m}$ and a variety of scaffolding densities.

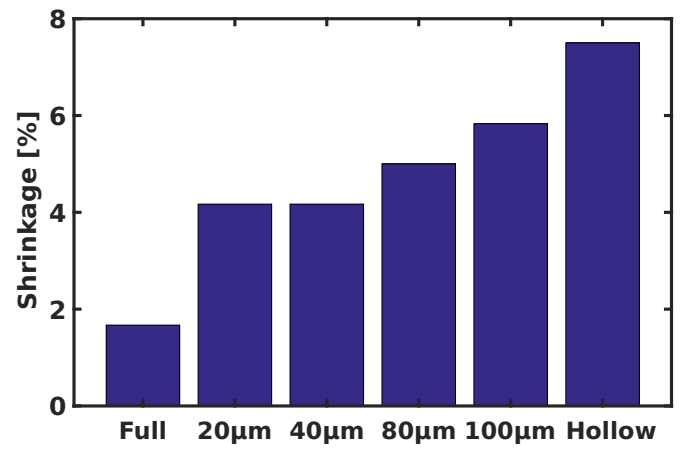

Figure 10: Shrinkage of the test structures of Figure 10 after flood exposure

\subsection{Measurements}

A chip sample was measured prior and after packaging with a horn antenna. Figure 11 shows the measurement setup. The transmitter is placed at a distance of around $10 \mathrm{~cm}$ from a VDI SAX module. The distance should be about 10 times greater then the maximum dimension of the antenna to ensure far field radiation characteristics. The VDI SAX module is a sub-harmonic mixer that downconverts the $400 \mathrm{GHz}$ signal to a low IF signal $(15 \mathrm{kHz}-2.5 \mathrm{GHz})$ which is then connected to an R\&S FSU spectrum analyzer. The spectrum analyzer can then be used to determine the frequency and radiating power of the radiation chip. Figure 12 shows the received power before and after packaging the transmitter. The bias voltage of the chip is sweeped, thus changing the radiating power and frequency. The red line shows the unpackaged chip and the blue line shows the same chip after packaging it with a horn antenna made through the method of section 3.1. A difference in received power of $12 \mathrm{~dB}$ is observed. Note that the received power in the spectrum analyzer is not equal to the output power of the 
transmitter. De radiated waves undergo free-space-path loss and conversion loss from the VDI-SAX before being measured by the spectrum analyzer. The performance of the packaged system is comparable to a system with silicon lenses.

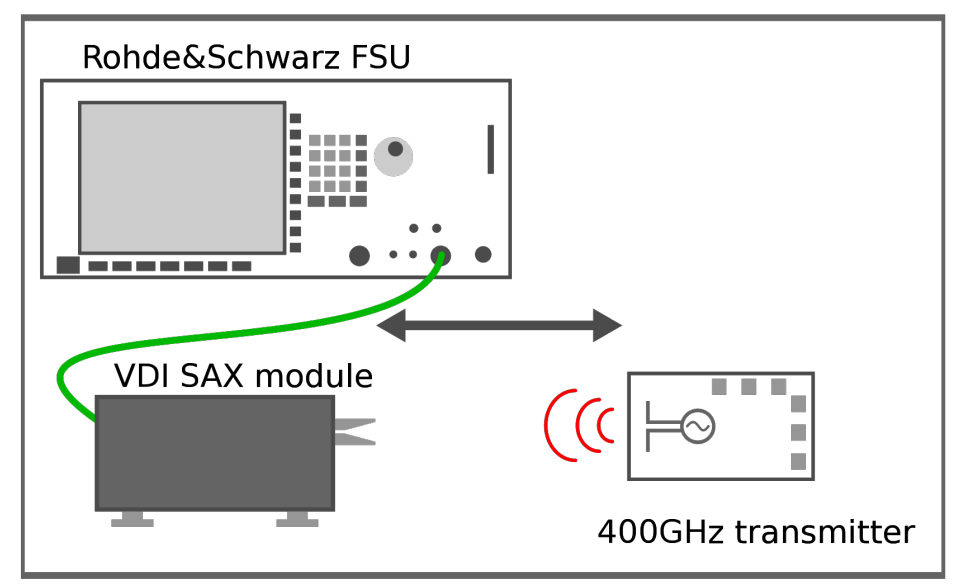

Figure 11: Measurement Setup: The CMOS chip is placed in front of a sub-harmonic mixer. The output of the mixer is connected to a Spectrum analyzer, from which the received power is determined

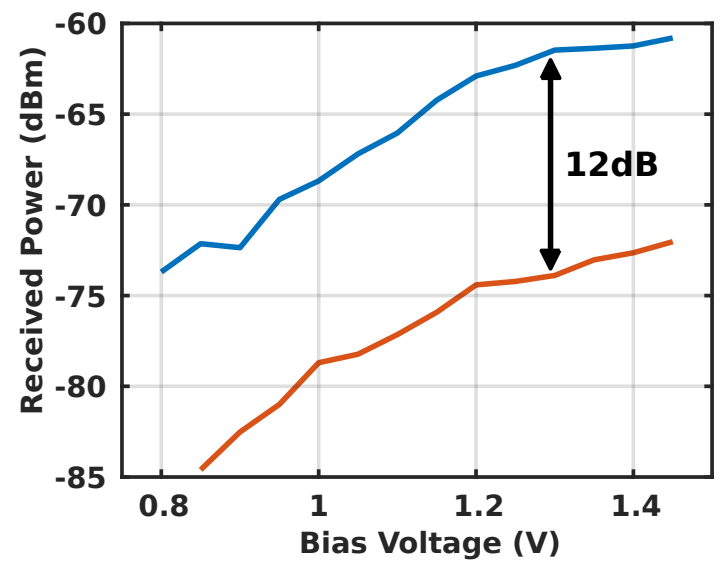

Figure 12: Received power on the spectrum analyzer in function of the bias voltage of the $400 \mathrm{GHz}$ transmitter. The red line shows the results of the unpackaged chip. The blue line shows the results of the packaged chip with a horn antenna fabricated with the method of section 3.1. A difference of received power of $12 \mathrm{~dB}$ is observed. 
Table 3: COMPARISON WITH EXISTING TECHNOLOGIES

\begin{tabular}{|l||c|c|c|l|}
\hline & Cost & Scalability & Integration & Design flexibility \\
\hline \hline $\begin{array}{l}\text { Two-photon lithography } \\
\text { (this work) }\end{array}$ & moderate & difficult & high & Very high \\
\hline Replica molding (this work) & low & easy & high & high (dependent on mold) \\
\hline Electroforming (this work) & low & easy & high & high \\
\hline $\begin{array}{l}\text { Metallic split-block waveg- } \\
\text { uides }\end{array}$ & high & difficult & medium & medium \\
\hline LTCC & low & easy & high & limited to 2.5D shapes \\
\hline Silicon lenses & moderate & medium & high & low \\
\hline
\end{tabular}

\subsection{Discussion and Comparison}

The methods described in this paper show some advantages and disadvantages compared to other existing technologies used for the integration of mm-wave and $\mathrm{THz}$ circuits. The biggest advantage is the design flexibility: The developed technology makes it possible to make fully 3D shapes without the need to take into account tool sizes as is the case with metallic split-block waveguides[6][7] or substrate height as is the case with LTCC[8][9]. The replica molding and electroforming solutions show promise in being scalable, enabling large volume manufacturing at low cost just as is the case with LTCC. As the proposed solutions interact directly with the CMOS chip, there is no need for high frequency transitions on expensive substrates as is the case with metallic splitblock waveguides. This results in a high level of integration as is the case with silicon lenses[10][11][27]. The biggest limiting factor in the proposed manufacturing processes in the metalisation with dealing with overhanging shapes. The Electroforming partially solves this but future work would benefit with an electroless plating like in [28],[29] or an ALD processing step to deposit a conductive seed layer. Table 3 shows a comparison between the developed and existing technologies used for the integration of mm-wave and $\mathrm{THz}$ circuits.

\section{Conclusion}

Three different methods were presented to fabricate horn antennas for millimeter-wave and terahertz frequencies. A first method using two-photon lithography provides a highly flexible and precise way of fabricating prototypes. A second method using a molding technique allows a quick replication of prototypes. A third method of fabrication, relying on an electroforming technique allows to make narrow high aspect ration waveguide sections. The prototypes were analyzed on their fabrication accuracy. The greatest source of errors was found to be the shrinkage of the polymer during flood exposure. This could be reduced by increasing the scaffolding density. A prototype was accurately positioned on top of a $400 \mathrm{GHz}$ CMOS transmitter and glued into place thus presenting a fully packaged solution. Measurements show an increase of antenna gain of $12 \mathrm{~dB}$. The suggested solution to package high frequency chip provides a interesting and 
valid alternative to the existing packaging solutions. The presented fabrication methods are not limited to manufacturing horn antennas and can be used to make a variety of high precision mm-sized metal components.

\section{Acknowledgement}

This work was supported by the Hercules Foundation for heavy equipment (ZW1115) and by the CHIST-ERA project "WISDOM" under FWO grant G0H5816N.

\section{References}

[1] Wang C and Han R 2017 17.6 Rapid and energy-efficient molecular sensing using dual mm-Wave combs in 65nm CMOS: A 220-to-320GHz spectrometer with $5.2 \mathrm{~mW}$ radiated power and 14.6-to19.5dB noise figure 2017 IEEE International Solid-State Circuits Conference (ISSCC) (IEEE) pp 302-303

[2] Chi T, Huang M Y, Li S and Wang H 201717.7 A packaged 90-to-300GHz transmitter and 115to-325GHz coherent receiver in CMOS for full-band continuous-wave mm-wave hyperspectral imaging 2017 IEEE International Solid-State Circuits Conference (ISSCC) (IEEE) pp 304-305

[3] Steyaert W and Reynaert P 2014 IEEE Journal of Solid-State Circuits 49 1617-1626

[4] Dhillon S S, Vitiello M S, Linfield E H, Davies A G, Hoffmann M C, Booske J, Paoloni C, Gensch M, Weightman P, Williams G P, Castro-Camus E, Cumming D R S, Simoens F, EscorciaCarranza I, Grant J, Lucyszyn S, Kuwata-Gonokami M, Konishi K, Koch M, Schmuttenmaer C A, Cocker T L, Huber R, Markelz A G, Taylor Z D, Wallace V P, Zeitler J A, Sibik J, Korter T M, Ellison B, Rea S, Goldsmith P, Cooper K B, Appleby R, Pardo D, Huggard P G, Krozer V, Shams H, Fice M, Renaud C, Seeds A, Sthr A, Naftaly M, Ridler N, Clarke R, Cunningham J E and Johnston M B 2017 Journal of Physics D: Applied Physics 50043001 URL http://stacks.iop.org/0022-3727/50/i=4/a=043001

[5] Song H J 2017 Proceedings of the IEEE 105 1121-1138 ISSN 0018-9219

[6] Bruneau P J, Janzen H D and Ward J S 2008 Machining of terahertz split-block waveguides with micrometer precision 2008 33rd International Conference on Infrared, Millimeter and Terahertz Waves pp 1-2

[7] Schur J, Biber S, Cojocari O, Schmidt L P and Hartnagel H L 2005600 ghz gaas schottky diode mixer in split-block technology 2005 Joint 30th International Conference on Infrared and Millimeter Waves and 13th International Conference on Terahertz Electronics vol 2 pp 469-470 vol. 2

[8] Tajima T, Song H J and Yaita M 2016 IEEE Transactions on Microwave Theory and Techniques 64 106-114

[9] Tajima T, Song H J, Ajito K, Yaita M and Kukutsu N 2014 IEEE Transactions on Antennas and Propagation 62 5437-5444

[10] Pfeiffer U R, Zhao Y, Grzyb J, Al Hadi R, Sarmah N, Forster W, Rucker H and Heinemann B 2014 IEEE Journal of Solid-State Circuits 49 2938-2950 ISSN 0018-9200

[11] Han R and Afshari E 2013 IEEE Journal of Solid-State Circuits 48 3090-3104 ISSN 0018-9200

[12] Ikawa-Smith F 1993 International Progress in Precision Engineering: Proceedings of the 7th International Precision Engineering Seminar, Kobe, Japan, May 1993 (Elsevier Science) ISBN 9780124158986

[13] Balanis C A 2005 Antenna Theory: Analysis and Design, 3rd Edition 3rd ed (Wiley-Interscience) ISBN 047166782X

[14] Guney M G and Fedder G K 2016 Journal of Micromechanics and Microengineering 26105011 URL http://stacks.iop.org/0960-1317/26/i=10/a=105011 
[15] En-Shinn Wu James H Strickler W R H W W W 1992 Two-photon lithography for microelectronic application vol 1674 pp $1674-1674-7$

[16] Park S, Yang D and Lee K 2009 Laser $\&$ Photonics Reviews 3 1-11 ISSN 1863-8899 URL http://dx.doi.org/10.1002/lpor.200810027

[17] Maruo S and Fourkas J 2008 Laser 83 Photonics Reviews 2 100-111 ISSN 1863-8899 URL http://dx.doi.org/10.1002/lpor.200710039

[18] Guney M G and Fedder G K 2016 Journal of Micromechanics and Microengineering 26105011 ISSN 0960-1317

[19] Ip-photoresist datasheet www.nanoscribe.de/files/1814/0662/4393/IP-Resist_IP-Dip_web. pdf

[20] Koroleva A, Gill A A, Ortega I, Haycock J W, Schlie S, Gittard S D, Chichkov B N and Claeyssens F 2012 Biofabrication 4025005 URL http://stacks.iop.org/1758-5090/4/i=2/a=025005

[21] Sammoura F, Fuh Y K and Lin L 2008 Journal of Micromechanics and Microengineering 18055009 ISSN 0960-1317 URL http://stacks . iop.org/0960-1317/18/i=5/a=055009?key=crossref . c8e8b2bf19586e9923011cce606456b2

[22] Sun H, Wang X, Xiong Y, Liu G and Wang K 2016 Journal of Micromechanics and Microengineering 2655007 URL http://stacks.iop.org/0960-1317/26/i=5/a=055007

[23] Banham R, Valsecchi G, Lucci L, Pelosi G, Selleri S, Natale V, Nesti R and Tofani G 2005 MICROWAVE JOURNAL 48 112+ ISSN 0192-6225

[24] Raman K, Rajagopalan S R and Siddiqi M I A 1964 Journal of Scientific Instruments 41340 URL http: //stacks.iop.org/0950-7671/41/i=5/a=437

[25] Malekabadi A and Paoloni C 2016 Journal of Micromechanics and Microengineering 26095010 URL http://stacks.iop.org/0960-1317/26/i=9/a=095010

[26] Totten G and MacKenzie D 2003 Handbook of Aluminum: Volume 2: Alloy Production and Materials Manufacturing Handbook of Aluminum (CRC Press) ISBN 9780203912607

[27] Guo K and Reynaert P 2017 A 475-511ghz radiating source with siw-based harmonic power extractor in $40 \mathrm{~nm}$ cmos 2017 IEEE MTT-S International Microwave Symposium (IMS) pp 95-98

[28] Ikegami T, Stocker M P, Monaco K, Fourkas J T and Maruo S 2012 Japanese Journal of Applied Physics 51 06FL17 URL http://stacks.iop.org/1347-4065/51/i=6S/a=06FL17

[29] Mukai K, Yoshimura T and Maruo S 2007 Japanese Journal of Applied Physics 462761 URL http://stacks. iop.org/1347-4065/46/i=4S/a=2761 